\title{
Non-locality and time-dependent boundary conditions: a Klein-Gordon perspective
}

\author{
S. Colin and A. Matzkin \\ Laboratoire de Physique Théorique et Modélisation, \\ CNRS Unité 8089, CY Cergy Paris Université, \\ 95302 Cergy-Pontoise cedex, France
}

\begin{abstract}
The dynamics of a particle in an expanding cavity is investigated in the Klein-Gordon framework in a regime in which the single particle picture remains valid. The cavity expansion represents a time-dependent boundary condition for the relativistic wavefunction. We show that this expansion induces a non-local effect on the current density throughout the cavity. Our results indicate that a relativistic treatment still contains apparently spurious effects traditionally associated with the unbounded velocities inherent to non-relativistic solutions obtained from the Schrödinger equation. Possible reasons for this behaviour are discussed.
\end{abstract}

\section{INTRODUCTION}

Every prediction we can make in a quantum system is encoded in the quantum state, which is generally not localized, but extended over all the available space. The quantum state evolves deterministically by way of an evolution equation: the Schrödinger equation in non-relativistic quantum mechanics, the Klein-Gordon or Dirac equations in single particle relativistic quantum mechanics. Because the quantum state is extended all over space, it has features that link together instantaneously properties in different regions of space. However this non-locality cannot be used to communicate. If signaling were possible, we could communicate with the future and modify the past. It turns out however that the Schrödinger equation displays in some cases signaling [1, 2]. This is not necessarily worrying because the Schrödinger equation being non-relativistic there is no bound on the velocity associated with its solutions. But sometimes it is not obvious to assert whether this form of signaling is an artifact (employing a non-relativistic equation), or is a genuine feature of quantum non-locality.

A concrete example was investigated in [3] where the authors considered the case of a non-relativistic particle placed in a one-dimensional cavity whose right wall can be set in uniform motion. This system - an infinite well with a moving wall - has often been suspected of displaying some form of single-particle non-locality 47] and has very recently been found to be relevant in atomic 8, 9] or neutron [10 spectroscopy. Assume a particle is prepared in a given state of a cavity for which the right wall can either be static or in motion (the cavity is then in expansion). At $t=0$, the length of the cavity is $L(t=0)=L_{0}$. The current $j(t, x)$ at $x=\epsilon$, where $\epsilon$ lies near the left wall (the side opposite to the wall that is allowed to move) will be different in both cases. As shown in [3, the current can in principle be measured by performing a weak measurement (as will be recalled below). It was found that the current value is non-local, in the sense that the current difference $\Delta j$ between both cases obeys $\Delta j(t, \epsilon) \neq 0$ for $t<\frac{\left|L_{0}-\epsilon\right|}{c}$ where $c$ is the light velocity. This can lead to a protocol based on monitoring the current by which it is possible to send information faster-than-light. However the results reported in [3] were obtained with solutions of the Schrödinger equation. Indeed, solutions of the Schrödinger equation with a moving wall have been known analytically for some time for specific wall motions [11, 12, But since the Schrödinger equation is non-relativistic, any expansion over the energy eigenstates will formally include states associated with arbitrarily high energies, corresponding to supraluminal velocities. The question is therefore whether the supraluminal results constitute an artifact of the description of the system by the Schrödinger equation.

The goal of the present article is precisely to answer this question by investigating the same system in a relativistic setting based on the Klein-Gordon (KG) equation. We will rely on the solutions of the KG equation for a particle inside a uniformly expanding cavity that were recently obtained 13 15. Note that several properties of single-particle relativistic wave equations have been investigated recently [16-19]. As is well-known, the $\mathrm{KG}$ equation is not free of interpretation problems, but we will consider a regime in which these problems do not appear.

The structure of the paper is as follows. We first recall the solutions for the KG equation with a boundary condition expanding linearly in time that were obtained recently [13 15]. We also derive expressions for the current density. We show that the relativistic wavefunctions and current density reduce to the non-relativistic solutions used in 3 in the limit where the wall is slowly expanding and the length of the cavity is large with respect to the Compton wavelength of the particle. We then compare the currents as a function of time when the outer wall is moving or remains fixed. We will see that this current change appears instantaneously inside the cavity. We examine whether artifacts could account for these results and discuss how a transient regime could be included in the model. We finally present our conclusions concerning single-particle non-locality in the relativistic case. 


\section{KG EQUATION WITH A MOVING BOUNDARY}

\section{A. Analytical solutions for a KG particle inside a linearly expanding cavity}

A KG particle of mass $m$ is initially trapped in a fixed infinite well (the potential is equal to zero for $x \in\left[0, L_{0}\right]$ and to $+\infty$ elsewhere). The solutions of the KG equation

$$
\frac{\partial_{t}^{2} \Phi}{c^{2}}-\partial_{x}^{2} \Phi+\frac{m^{2} c^{2}}{\hbar^{2}} \Phi=0
$$

are given by

$$
\Phi_{ \pm, n}(t, x)=\frac{1}{\sqrt{2 E_{n} L_{0}}} e^{i \pm \frac{E_{n} t}{\hbar}} \sin \left(\frac{p_{n} x}{\hbar}\right)
$$

with $n=1,2,3, \ldots, p_{n}=\frac{\hbar n \pi}{L_{0}}$ and $E_{n}=\sqrt{m^{2} c^{4}+p_{n}^{2} c^{2}}$. The $+/-$ index refers to the signs in the exponent, opposite to the sign of the energies $(+/-$ refers to antiparticles/particles respectively). These solutions are orthonormal with respect to the KG scalar product 1

$$
(\Phi, \Xi)_{K G}=\int d x\left(\Phi^{*} i \hbar \partial_{t} \Xi-i \hbar\left(\partial_{t} \Phi^{*}\right) \Xi\right)
$$

the KG probability density for a given state $\Phi$ being defined as

$$
\rho_{K G}(t, x)=\left(\Phi^{*} i \hbar \partial_{t} \Phi-i \hbar\left(\partial_{t} \Phi^{*}\right) \Phi\right) .
$$

At $t=0$, the right wall starts to move with constant velocity $v=\beta c$ and its position at time $t$ is therefore given by $L=L_{0}+v t$ (the left wall remains fixed). The analytical solutions of this problem (a KG particle in an infinite square-well potential with a linearly expanding wall) were obtained by Koehn (see eq. 11 in [13); other authors proposed a generalization shortly after [14], and gave an alternative method in [15. These solutions can be written as linear superpositions of

$$
\Psi_{J, n}=N_{J, n} J_{i k_{n}}\left(\frac{\sqrt{L^{2}-\beta^{2} x^{2}}}{\lambda_{C} \beta}\right) \sin \left(\frac{k_{n}}{2} \ln \left(\frac{L+\beta x}{L-\beta x}\right)\right)
$$

and

$$
\Psi_{Y, n}=N_{Y, n} Y_{i k_{n}}\left(\frac{\sqrt{L^{2}-\beta^{2} x^{2}}}{\lambda_{C} \beta}\right) \sin \left(\frac{k_{n}}{2} \ln \left(\frac{L+\beta x}{L-\beta x}\right)\right)
$$

\footnotetext{
${ }^{1}$ Recall (see eg Ch 1 of Ref. 20]) that since the KG equation is relativistic the continuity equation $\partial_{\mu} j^{\mu}=0$ involves the 4vector $j^{\mu} \equiv \frac{i \hbar}{2 m}\left(\psi^{*} \partial^{\mu} \psi-\psi \partial^{\mu} \psi^{*}\right)$ whose time component is the density $j^{0}=\rho c$ while the space component is the current familiar from non-relativistic quantum mechanics; we set $\left(x^{\mu}\right)=(c t, \mathbf{x})$ for $\mu=0$ and $\mu=1,2,3$ resp. and $x^{\mu} x_{\mu}=c^{2} t^{2}-\mathbf{x}^{2}$. Hence normalization of the density $\int d x \rho(x)$ implies the integrand is $\psi^{*} \partial_{t} \psi-\psi \partial_{t} \psi^{*}$. This is an indication that the scalar product is given by Eq. (3). The presence of the time derivative is due to the fact that the KG equation is second order in time (for a full account see [21]).
}

where $\lambda_{C}=\frac{\hbar}{m c}$ (the Compton wavelength of the $\mathrm{KG}$ particle), $k_{n}=\frac{2 n \pi}{\ln \left(\frac{1+\beta}{1-\beta}\right)}$ with $n=1,2,3, \ldots, N_{J, n}$ and $N_{Y, n}$ being normalization constants. $J$ and $Y$ are Bessel functions respectively regular and irregular at the origin [24. Alternatively, one can use the basis of solutions

$$
\Psi_{-, n}=N_{J, n} J_{-i k_{n}}\left(\frac{\sqrt{L^{2}-\beta^{2} x^{2}}}{\lambda_{C} \beta}\right) \sin \left(\frac{k_{n}}{2} \ln \left(\frac{L+\beta x}{L-\beta x}\right)\right)
$$

and

$$
\Psi_{+, n}=N_{J, n} J_{i k_{n}}\left(\frac{\sqrt{L^{2}-\beta^{2} x^{2}}}{\lambda_{C} \beta}\right) \sin \left(\frac{k_{n}}{2} \ln \left(\frac{L+\beta x}{L-\beta x}\right)\right),
$$

with $\Psi_{+, n}=\Psi_{J, n}$.

\section{B. The non-relativistic limit}

We will now assume $\beta \ll 1$ and $\beta x \ll L$. Indeed typically (in particular if we have experiments in mind) the wall motion will be non-relativistic, and recall, as mentioned in the Introduction, that we will be interested in values of the current density near the fixed wall. Thus the orders of the Bessel functions of interest will be very large in magnitude, with $k_{n} \approx \frac{n \pi}{\beta}$, and the sine part of the above solutions can be approximated as

$$
\sin \left(\frac{k_{n}}{2} \ln \left(\frac{L+\beta x}{L-\beta x}\right)\right) \approx \sin \left(k_{n} \beta \frac{x}{L}\right) \approx \sin \left(n \pi \frac{x}{L}\right) .
$$

To keep the expressions short in the following, we will use

$$
\phi_{n}=\frac{k_{n}}{2} \ln \left(\frac{L+\beta x}{L-\beta x}\right) .
$$

The argument of the Bessel functions, denoted $z$ for short,

$$
z \equiv \frac{\sqrt{L^{2}-\beta^{2} x^{2}}}{\lambda_{C} \beta},
$$

can also be very large (firstly $\beta$ is small, secondly $L_{0} \gg$ $\left.\lambda_{C}\right)$. Therefore we will consider the approximation for the Bessel functions of imaginary order in the limit where the argument is large and positive.

For that purpose, the functions $\tilde{J}_{\nu}(z)$ and $\tilde{Y}_{\nu}(z)$ (with real $\nu$ and positive $z$ ) are introduced (see 22] section 3 for a starting point, then [23] and [24]) as

$$
\tilde{J}_{\nu}=\operatorname{sech}\left(\frac{\pi}{2} \nu\right) \Re\left(J_{i \nu}\right), \quad \tilde{Y}_{\nu}=\operatorname{sech}\left(\frac{\pi}{2} \nu\right) \mathfrak{R}\left(Y_{i \nu}\right) .
$$

Thanks to the relations

$$
\mathfrak{R}\left(Y_{i \nu}\right)=\operatorname{coth}\left(\frac{\pi}{2} \nu\right) \mathfrak{I}\left(J_{i \nu}\right), \quad \mathfrak{I}\left(Y_{i \nu}\right)=-\tanh \left(\frac{\pi}{2} \nu\right) \mathfrak{R}\left(J_{i \nu}\right),
$$


the functions $J$ and $Y$ can be expressed as

$$
J_{i \nu}(z)=\cosh \left(\frac{\pi}{2} \nu\right) \tilde{J}_{\nu}(z)+i \sinh \left(\frac{\pi}{2} \nu\right) \tilde{Y}_{\nu}(z)
$$

and

$$
Y_{i \nu}(z)=\cosh \left(\frac{\pi}{2} \nu\right) \tilde{Y}_{\nu}(z)-i \sinh \left(\frac{\pi}{2} \nu\right) \tilde{J}_{\nu}(z) .
$$

Employing the asymptotic expansions given in [23] (see eqs. (3.17) and (3.18) of [23] details are given in the Appendix) to second order, we obtain

$$
\begin{aligned}
& \tilde{J}_{\nu}(z) \approx \sqrt{\frac{2}{\pi z}}\left(\cos \left(z-\frac{\pi}{4}\right)+\frac{4 \nu^{2}+1}{8 z} \sin \left(z-\frac{\pi}{4}\right)\right) \\
& \tilde{Y}_{\nu}(z) \approx \sqrt{\frac{2}{\pi z}}\left(\sin \left(z-\frac{\pi}{4}\right)-\frac{4 \nu^{2}+1}{8 z} \cos \left(z-\frac{\pi}{4}\right)\right) .
\end{aligned}
$$

Using these forms in Eq. (14), we obtain the following expressions

$$
\begin{aligned}
J_{i \nu}(z) & \approx \cosh \left(\frac{\pi}{2} \nu\right) \sqrt{\frac{2}{\pi z}}\left(e^{i\left(z-\frac{\pi}{4}\right)}\left[1-i \frac{4 \nu^{2}+1}{8 z}\right]\right) \\
& \approx \cosh \left(\frac{\pi}{2} \nu\right) \sqrt{\frac{2}{\pi z}}\left(e^{i\left(z-\frac{4 \nu^{2}+1}{8 z}-\frac{\pi}{4}\right)}\right),
\end{aligned}
$$

and the analytical solutions (Eqs. (8) and (7) become

$$
\Psi_{+, n} \approx N_{+, n} \cosh \left(\frac{\pi}{2} k_{n}\right) \sqrt{\frac{2}{\pi z}} e^{i\left(z-\frac{4 k_{n}^{2}+1}{8 z}-\frac{\pi}{4}\right)} \sin \left(\phi_{n}\right)
$$

and

$$
\Psi_{-, n} \approx N_{-, n} \cosh \left(\frac{\pi}{2} k_{n}\right) \sqrt{\frac{2}{\pi z}} e^{-i\left(z-\frac{4 k_{n}^{2}+1}{8 z}-\frac{\pi}{4}\right)} \sin \left(\phi_{n}\right) .
$$

Now let us show that the last expression 20, corresponding to particles (positive energies) indeed reduces to the solution of the Schrödinger equation given by [3, 12 .

$$
\psi_{n}(t, x)=\sqrt{\frac{2}{L}} \exp \left(-\frac{i \pi^{2} \hbar n^{2} t}{2 m L_{0} L}\right) \exp \left(\frac{i m v x^{2}}{2 \hbar L}\right) \sin \left(\frac{n \pi x}{L}\right) .
$$

First we note that the factor $\sqrt{\frac{2}{\pi z}} \approx \sqrt{\frac{2}{\pi L}}$ when $\beta \ll 1$ (justifying the factor $\sqrt{\frac{2}{L}}$ in $\sqrt{21}$.) Secondly we have already pointed out that $\sin \left(\phi_{n}\right) \approx \sin \left(\frac{n \pi x}{L}\right)$ (hence the presence of $\sin \left(\frac{n \pi x}{L}\right)$ in $\left.(21)\right)$. Finally the imaginary exponential term $z-\frac{4 \nu^{2}+1}{8 z}$ becomes in that limit $\frac{m c^{2} t}{\hbar}-\frac{m v x^{2}}{2 \hbar L}+\frac{\hbar n^{2} \pi^{2} t}{2 m L_{0} L}$ (the proof is given in the Appendix).

\section{Normalization}

We want to compute the KG density (4) for the state

$$
\Psi_{-, n}(t, x)=N_{-, n} J_{-i k_{n}}(z) \sin \left(\phi_{n}\right) \text {. }
$$

We first note that the following relation

$$
\frac{d J_{-i \nu}(z)}{d z}=\frac{1}{2}\left(J_{-i \nu-1}(z)-J_{-i \nu+1}(z)\right)
$$

holds although the orders of the Bessel functions are imaginary. Then, from the definition of the density (4), the previous relation and the following ones

$$
\begin{array}{r}
\partial_{t} z=\frac{c}{\lambda_{C}} \frac{L}{\sqrt{L^{2}-\beta^{2} x^{2}}}=\frac{c L}{\beta \lambda_{C}^{2} z}, \\
\partial_{t} \phi_{n}=\frac{-k_{n} \beta v x}{L^{2}-\beta^{2} x^{2}}=-\frac{k_{n} c x}{\lambda_{C}^{2} z^{2}},
\end{array}
$$

obtained from (11) and 10 , we find the density

$$
\rho_{K G}(t, x)=N_{-, n}^{2} \hbar \sin ^{2}\left(\phi_{n}\right) \frac{\partial z}{\partial t}\left[2 \mathfrak{I m}\left(J_{-i k_{n}}(z) \frac{d J_{i k_{n}}(z)}{d z}\right)\right] .
$$

In the non-relativistic limit, the above expression can be further approximated to

$$
\rho_{K G}(t, x)=N_{-, n}^{2} \hbar \sin ^{2}\left(\phi_{n}\right)\left(\frac{c L}{\beta \lambda_{C}^{2} z}\right)\left[\frac{4}{\pi z}\left(\frac{1}{\cosh \frac{\pi k_{n}}{2}}\right)^{2}\right] .
$$

Using $z \approx \beta^{-1} \lambda_{C}^{-1} L$, we find that

$$
\int d x \rho_{K G}(t, x)=N_{-, n}^{2} \frac{2 \hbar c \beta}{\pi}\left(\frac{1}{\cosh \frac{\pi k_{n}}{2}}\right)^{2} .
$$

Therefore, if we introduce the $C_{ \pm, n}$ thanks to $N_{ \pm, n}=$ $C_{ \pm, n} \sqrt{\frac{\pi}{2 \beta c \hbar}} \frac{1}{\cosh \left(\frac{\pi k_{n}}{2}\right)}$, the normalized states will read

$$
\Psi_{ \pm, n}(t, x)=C_{ \pm, n} \sqrt{\frac{\pi}{2 \beta c \hbar}} \frac{1}{\cosh \left(\frac{\pi k_{n}}{2}\right)} J_{ \pm i k_{n}}(z) \sin \left(\phi_{n}\right)
$$

and we will have that $C_{ \pm, n} \approx 1$ in the non-relativistic limit.

\section{Current density}

The KG current density for a given state $\Phi$ is defined as

$$
j_{K G}(t, x)=-\hbar c^{2}\left(\Phi^{*} i \partial_{x} \Phi-i\left(\partial_{x} \Phi^{*}\right) \Phi\right) .
$$

Its computation for the state $\Psi_{-, n}(t, x)$ is not much different from that of the KG probability density. First we have that

$$
\begin{array}{r}
\partial_{x} z=-\frac{1}{\lambda_{C}} \frac{\beta x}{\sqrt{L^{2}-\beta^{2} x^{2}}}=-\frac{x}{\lambda_{C}^{2} z}, \\
\partial_{x} \phi_{n}=\frac{k_{n} \beta L}{L^{2}-\beta^{2} x^{2}}=\frac{k_{n} L}{\beta \lambda_{C}^{2} z^{2}} .
\end{array}
$$


Next, thanks to 23, 28) and 230, 29) becomes

$$
\begin{array}{r}
-C_{-, n}^{2} \frac{\pi}{2 \beta c \hbar} \frac{1}{\cosh ^{2}\left(\frac{\pi k_{n}}{2}\right)} \times \\
c^{2} \hbar \sin ^{2}\left(\phi_{n}\right) \frac{\partial z}{\partial x}\left[2 \mathfrak{I m}\left(J_{-i k_{n}}(z) \frac{d J_{i k_{n}}(z)}{d z}\right)\right] .
\end{array}
$$

In the non-relativistic limit, the last expression becomes

$$
j_{K G}(t, x) \approx 2 C_{-, n}^{2} \frac{x \beta c}{L^{2}} \sin ^{2}\left(\frac{n \pi x}{L}\right),
$$

which is the expression found in $\left[3\right.$, provided $C_{-, n}^{2} \rightarrow 1$.

\section{NON-LOCALITY IN THE KG EXPANDING CAVITY}

\section{A. Current change}

We examine here the effect of the expanding wall on the current density at the opposite side of the cavity. In order to do so, we assume the cavity length can either be fixed (the length is denoted by $L_{0}$ ), or in expansion, with the right wall moving at uniform velocity as in the previous Section. In both cases, the KG particle is prepared in the state $\Psi_{-, 1}(t=0, x)$, and the cavity length is tuned such that ${ }^{2} L(t=0)=L_{0}$. In the case the cavity is expanding, we know the later current to be given by Eq. $29 p$. We then compare this current $\left(j_{e}\right)$ to the one $\left(j_{s}\right)$ corresponding to the situation in which the well is static. $j_{s}$ is obtained by expanding $\Psi_{-, 1}(0, x)$ on the energy eigenstates of the fixed-walls cavity

$$
\Psi_{-, 1}(t=0, x)=\sum_{n} c_{n} \Phi_{-, n}(t=0, x)
$$

where $\Phi_{-, n}$ is given by Eq. (2) and

$$
\begin{aligned}
c_{n} & =\left(\Phi_{-, n}, \Psi_{-, 1}\right)_{K G} \\
& =\int d x\left(\Phi_{-, n}^{*} i \hbar \partial_{t} \Psi_{-, 1}+E_{n} \Phi_{-, n}^{*} \Psi_{-, 1}\right) .
\end{aligned}
$$

At later times, the wavefunction is given by $\Psi_{-, 1}(t, x)$ when the wall is expanding and by $\sum_{n} c_{n} \Phi_{-, n}(t, x)$ for a stationary cavity. The resulting density currents $j_{e}$ and $j_{s}$ will therefore be different. Note that since we are in a non-relativistic regime, the anti-particles contribution are not expected to be significant, as we verify below. The next aspect to discuss is the choice of parameters and some related numerical aspects.

\footnotetext{
2 This tuning is chosen because we require the initial state to be the same in both cases. Note that the preparation procedure does not have to be identical for both configurations, the requirement is that the initial state is the same.
}

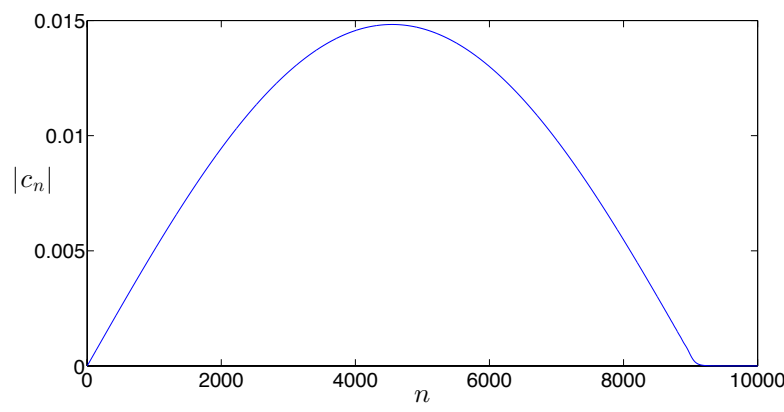

FIG. 1. The norm of the expansion coefficients $c_{n}$ (Eq. (34)) as a function of $n$. The maximum value is reached at $n=$ 4551. We have that $\left|c_{9000} / c_{4551}\right|=0.0312,\left|c_{10000} / c_{4551}\right|=$ $2.6148 \times 10^{-5}$ and $\left|c_{15000} / c_{4551}\right|=5.7616 \times 10^{-7}$.

\section{B. Numerical aspects}

To avoid any problem of interpretation of the KleinGordon equation, we work in the non-relativistic regime characterized by

$$
\beta \ll 1 \text { and } z \approx \frac{L_{0}}{\lambda_{C} \beta} \gg 1 .
$$

As explained in the Appendix, we cannot numerically use the approximations for the Bessel functions but need instead to rely on the exact forms. Since their values grow like $\cosh \left(\frac{\pi \nu}{2}\right) \propto e^{\frac{\pi \nu}{2}}$ with $\nu \approx n \pi / \beta, \beta$ cannot be too small in order to keep the computations tractable. For this reason, we will set $\beta=0.01$, which might be somewhat higher than a typical non-relativistic case but still abides by $\beta \ll 1$. We also need $z$ to be large, implying $L_{0} /\left(\beta \lambda_{C}\right)$ must be large.

\section{Example}

We consider the case

$$
m=10^{-30} \mathrm{~kg}, L_{0}=10^{-6} \mathrm{~m} \quad \beta=0.01 \text {, hence } z \approx 10^{8} .
$$

We assume that the initial state is $\Psi_{-, 1}(t=0, x)$. It can be expanded over the static cavity eigenstates as per Eq. (34). We can check that, as mentioned above, the anti-particles do not contribute: the scalar products $b_{n}=$ $\left(\Phi_{+, n}, \Psi_{-, 1}\right)_{K G}$ are negligible (we have found that $\left|b_{n}\right|<$ $10^{-14}$ for all $n<15000$ ). In Fig. 1 we plot the norm of the expansion coefficients $c_{n}$.

The presence probability density for $\Psi_{-, 1}(0, x)$ is almost identical to the one for $\Phi_{-, 1}(0, x)$ but contrary to $\Phi_{-, 1}(0, x), \Psi_{-, 1}(0, x)$ has a large number of internal oscillations depending on the system mass and cavity properties (see Fig 2 for a plot of its real part).

Then we compare both currents in a space-time region which is spacelike separated from the event $(t=0, x=$ $\left.L_{0}\right)$. This illustrated in Fig. 3 at $t=10^{-15}$ where the 


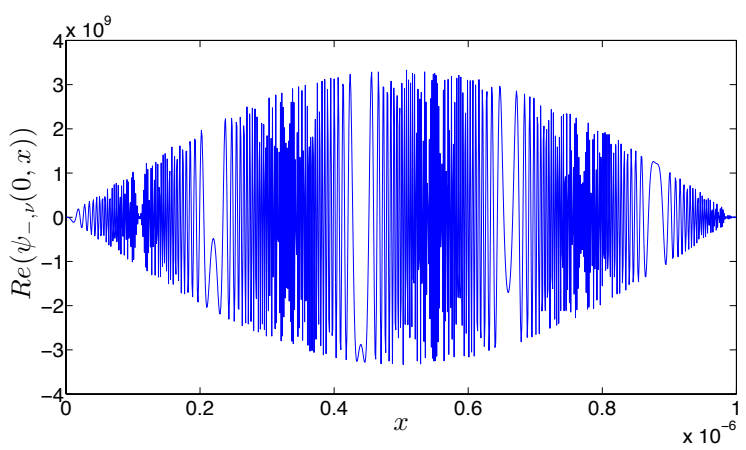

FIG. 2. A plot of the real part of $\Psi_{-, 1}(0, x)$ (Eq. (7)), intended to show the large number of internal oscillations (about 500). The imaginary part behaves in a similar way.

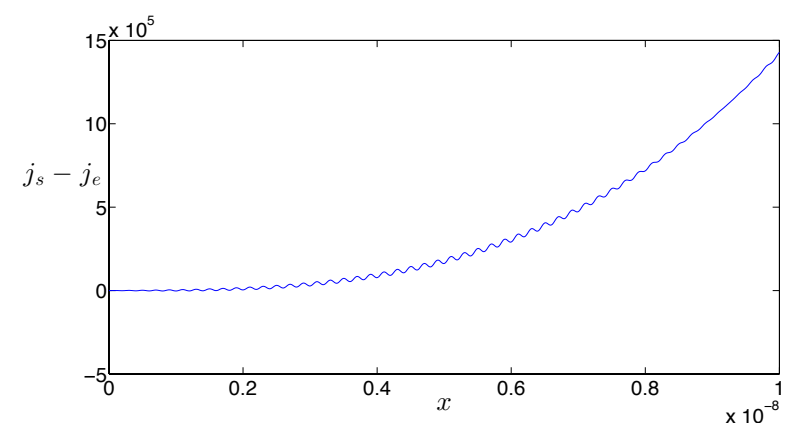

FIG. 3. Difference between the currents $j_{s}$ and $j_{e}$ at $t=$ $10^{-15} \mathrm{~s}$ in a region $\left[0,10^{-8} \mathrm{~m}\right]$ causally disconnected from the event $\left(0, L_{0}\right)$.

difference $j_{s}\left(t=10^{-15}, x\right)-j_{e}\left(t=10^{-15}, x\right)$ is plotted in a small region representing the leftmost $10^{-8} / 10^{-6}$ fraction of the cavity near the fixed wall. A light signal emitted from the right wall would take at least $\left(10^{-6}-\right.$ $\left.10^{-8}\right) / c=3.3 \times 10^{-15}$ seconds to reach this region.

For the figure, we have varied the number of steps for the numerical integration needed for the computation of the $c_{n}$ (the largest number of steps being 200000) and $n_{\max }$ (we have used 10000 and 15000); all runs gave almost identical curves. The relative difference between the currents $j_{e}$ and $j_{s}$ at $x=10^{-8} \mathrm{~m}$ is of the order of $2 \%$ while the relative difference between the box lengths at $t=0$ and $t=10^{-15} \mathrm{~s}$ is of the order of $0.3 \%$.

\section{DISCUSSION}

The present results confirm the non-local character of the quantum state in a relativistic setting: although the cavity is prepared in the same state at $t=0$, an observer located near the left wall $(x=0)$ can discriminate as soon as $t>0$ whether the right wall is static or expanding by monitoring the local current density. This does not depend on the cavity size, so the right wall movement is seen to affect the current density even if it lies in a space-like separated region (recall that the Hamiltonians in the static and moving cases are identical except in the region beyond $x=L_{0}$ ).

As argued in Ref. [3], this effect - if it is physical, as we discuss below - could in principle be used to communicate supraluminally. Indeed, the protocol employed in [3], based on weak measurements, remains essentially the same in the present case: a weak unitary interaction coupling the momentum of the particle in the cavity to a pointer takes place near $x \approx 0$. This unitary interaction is immediately followed by a measurement of the position at the same point. If the position measurement succeeds, the pointer has shifted by a quantity proportional to the real part of the weak value of the momentum $P^{w}$, defined by

$$
P^{w}=\frac{m j_{\psi}(t, x)}{|\psi(t, x)|^{2}}-i \hbar \frac{\partial_{x}\left(|\psi(t, x)|^{2}\right)}{2|\psi(t, x)|^{2}} .
$$

This definition of the weak value is characteristic of the non-relativistic formalism and cannot be extended straightforwardly to relativistic wavefunctions. However we are here in the non-relativistic regime, and we have shown above that $j_{\psi}(t, x) \approx j_{K G}(t, x)$ and $|\psi(t, x)|^{2} \approx$ $\rho_{K G}(t, x)$ (see Eqs. (32) and (21) resp.). So from an operational point of view, the weak measurement made on a relativistic particle in the non-relativistic regime will result in a shift proportional to $P^{w}$ and directly depending on the current density.

While there is no doubt that the present relativistic model gives rise to supraluminal communication and signaling, whether the model is physical can be questioned. The most obvious culprit in the non-relativistic framework was that the basis expansion akin to Eq. (33) in principle includes states with arbitrarily high energies (leading to arbitarily high velocities). This issue does not appear in our relativistic framework: by definition the velocity associated with the states in the expansion (33) is bounded by $c$. Actually in the numerical illustration we have given, we see from Fig. 1 that the expansion should include states up to $n \approx 10000$. Since by Eq. (2) $p_{n} \approx n \pi \hbar / L_{0}=m u / \sqrt{1-(u / c)^{2}}$ where $u$ is the velocity associated to the plane wave of momentum $p_{n}$, we have here $u / c \approx 1.1 \times 10^{-2}$ for $n=10000$.

Another possible artifact could come from the breakdown of the single particle picture that occurs when energies are sufficiently large so that particle creation becomes possible. We do not see any obvious reason to question the single particle picture here: first, the Klein paradox can be avoided in our infinite well [25]; second, as we have just seen, even the highest contributing energy eigenstate of the fixed wall cavity $(n=10000)$ yields a value for $p / m c \approx 10^{-2}$ reasonably below the particle creation threshold. A related issue is the apparent violation of causality for a Klein-Gordon particle when the initial state is tightly localized [26. It has been argued 27. that this violation, that would, as in our case, give rise to signaling, is not physical, as it requires exponential localization that is not compatible with solutions of 
wave equations such as the Klein-Gordon equation. In the present problem the particle is not tightly localized (the cavity can be arbitrarily long), and our observation relies on solutions of the KG equation.

Since there is no obvious artifact that could account for this relativistic non-local effect, it seems the model itself must be questioned. Indeed, the wavefunction, be it a relativistic wavefunction, is globally defined throughout configuration space - here throughout the entire cavity. If the potential changes at one end of the cavity, the wavefunction readjusts throughout the cavity instantaneously. It is well-known [28] that a sudden change in the potential or in the boundary conditions gives rise to a transient regime. The problem here is that we are not making an approximation that could be fixed in order to account for the transient regime (other than by including an ad-hoc prescription to account for retarded effects when the wall moves). Instead, it appears that the model itself needs to be modified: for instance we might need to model the moving wall otherwise than by a time-dependent infinite potential, for example by a field interacting with the system through exchange particles.
A quantum field treatment of the present problem would therefore be instructive.

\section{CONCLUSION}

To sum up, we have investigated a Klein-Gordon particle in an expanding cavity. We have shown that a curious form of single particle non-locality previously studied with the Schrödinger equation subsists in a relativistic setting. Contrary to the case computed with the nonrelativistic formalism, we have not identified any obvious artifacts that could account for the results. If we discard the possibility of this effect being physically real (given that this form of non-locality gives rise to signaling), our results lead to the conclusion that a relativistic model based on a local potential affecting a wavefunction defined over an extended region fails to capture correctly the dynamics. Investigating additional examples as well as a quantum field based treatment would be helpful in understanding the implications of the present results.
[1] Garcia-Calderon G., Rubio A.Villavicencio J., Phys. Rev. A 59 (1999), 1758.

[2] Ximenes R. Parisio F., Eur. Phys. J. Plus 131 (2016), 404.

[3] Matzkin A., Mousavi S. V. Waegell M., Phys. Lett. A 382 (2018), 3347.

[4] Greenberger D. M., Physica B 151 (1988), 374.

[5] Mousavi S. V., EPL 99 (2012), 30002.

[6] Matzkin A., J. Phys. A: Math. Theor. 51 (2018), 095303.

[7] Waegell M. Matzkin A., arXiv:1909.06465 (2019).

[8] Wang D., Phys. Rev. A 98 (2018) 053419.

[9] Duffin C. and Dijkstra A. G., Eur. Phys. J. D 73 (2019) 221.

[10] Pitschmann M. and Abele H., arXiv:1912.12236 (2019).

[11] Doescher S. W. Rice H. H., Am. J. Phys. 37 (1969) 1246.

[12] Makowski A. J. and Dembinski S. T., Phys. Lett. A 154 (1991), 217.

[13] Koehn M., EPL 100 (2012) 60008.

[14] Bialynicki-Birula I., EPL 101 (2013) 60003.

[15] Hamidi O. Dehghan H., Rep. Math. Phys. 73 (2014) 11.

[16] M. Aktas, EPL 121 (2018) 10005.

[17] L. Huang, X. H. Wu, T. Zhou, Sci. China-Phys. Mech. Astron. 61 (2018) 080311.

[18] Jun Feng et al, EPL 122 (2018) 60001.

[19] Dai-Nam Le et al, EPL 127 (2019) 10005.

[20] Greiner W., Relativistic Quantum Mechanics, Springer (1996).

[21] Mostafazadeh A., Ann. Phys. 309 (2004) 1.

[22] Chapman C. J., Proc. R. Soc. A 468 (2012) 4008.

[23] Dunster T. M., SIAM J. Math. Anal. 21 (1990) 995.

[24] Olver F. W. J., Asymptotics and Special Functions, Academic Press (1974).

[25] Alkhateeb M., Colin S. Matzkin A., in preparation.

[26] Hegerfeldt G. C., Phys. Rev. Lett. 72 (1994) 596.

[27] Barat N. Kimball J. C., Phys. Lett. A 310 (2003) 108.
[28] del Campo A., Garcia-Calderon G. Muga J. G., Phys. Rep. 476 (2009) 1.

\section{APPENDIX}

\section{KG Solutions : Asymptotic Expansion}

The functions $\tilde{J}_{\nu}(z)$ and $\tilde{Y}_{\nu}(z)$ given by Eq. 12 admit the following expansion (see Eqs. (3.17) and (3.18) of [23])

$$
\begin{aligned}
\tilde{J}_{\nu}(z)= & \sqrt{\frac{2}{\pi z}}\left(\cos \left(z-\frac{\pi}{4}\right) \sum_{s=0}^{s=\infty}(-1)^{s} \frac{A_{2 s}(i \nu)}{z^{2 s}}\right. \\
& \left.-\sin \left(z-\frac{\pi}{4}\right) \sum_{s=0}^{s=\infty}(-1)^{s} \frac{A_{2 s+1}(i \nu)}{z^{2 s+1}}\right)
\end{aligned}
$$

and

$$
\begin{aligned}
\tilde{Y}_{\nu}(z)= & \sqrt{\frac{2}{\pi z}}\left(\sin \left(z-\frac{\pi}{4}\right) \sum_{s=0}^{s=\infty}(-1)^{s} \frac{A_{2 s}(i \nu)}{z^{2 s}}\right. \\
& \left.+\cos \left(z-\frac{\pi}{4}\right) \sum_{s=0}^{s=\infty}(-1)^{s} \frac{A_{2 s+1}(i \nu)}{z^{2 s+1}}\right)
\end{aligned}
$$

where $A_{s}$ is defined as (see eq (4.02) of Ch. 7 in [24]):

$$
A_{s}(\nu)=\frac{\left(4 \nu^{2}-1\right)\left(4 \nu^{2}-3^{2}\right) \ldots\left\{4 \nu^{2}-(2 s-1)^{2}\right\}}{s ! 8^{s}} .
$$


These relations are useful as $z \rightarrow \infty$; then to lowest order, we obtain

$$
\tilde{J}_{\nu}(z) \approx \sqrt{\frac{2}{\pi z}} \cos \left(z-\frac{\pi}{4}\right) \text { and } \tilde{Y}_{\nu}(z) \approx \sqrt{\frac{2}{\pi z}} \sin \left(z-\frac{\pi}{4}\right) .
$$

Note that these expressions do not depend on $\nu$. Now we start from (5), we express the $J$ function in terms of $\tilde{J}$ and $\tilde{Y}$ (14) and we use the approximation A-4. After doing that, since $\cosh \left(\frac{\pi}{2} \nu\right) \approx \sinh \left(\frac{\pi}{2} \nu\right)$ for large $\nu$, we find that

$$
\begin{array}{r}
\Psi_{J, n} \approx N_{J, n} \cosh \left(\frac{\pi}{2} k_{n}\right) \sqrt{\frac{2 \beta \lambda_{C}}{\pi \sqrt{L^{2}-\beta^{2} x^{2}}}} \times \\
e^{+i\left(\frac{\sqrt{L^{2}-\beta^{2} x^{2}}}{\beta \lambda_{C}}-\frac{\pi}{4}\right)} \sin \left(\frac{n \pi x}{L}\right) \\
\approx N_{J, n} \cosh \left(\frac{\pi}{2} k_{n}\right) \sqrt{\frac{2 \beta \lambda_{C}}{\pi L}} e^{i \frac{L_{0}}{\beta \lambda_{C}}} \times \\
e^{+i \frac{m c^{2} t}{\hbar}} e^{-i \frac{\pi}{4}} \sin \left(\frac{n \pi x}{L}\right)
\end{array}
$$

(and similarly for $\Psi_{Y, n}$ ). Therefore this amounts to what is called the ultra non-relativistic limit. Note that it is best to use the functions $J_{i \nu}(z) \sin \left(\phi_{n}\right)$ and $J_{-i \nu}(z) \sin \left(\phi_{n}\right)$, also the basis used in [15], as they form the right basis for the emergence of non-relativistic solutions.

If we take the next terms in the series $(\mathrm{A}-1)$ and $\mathrm{A}-$ 2), we will obtain the standard non-relativistic limit. The approximations for (A-1) and (A-2) become

$$
\begin{aligned}
& \tilde{J}_{\nu}(z) \approx \sqrt{\frac{2}{\pi z}}\left(\cos \left(z-\frac{\pi}{4}\right)+\frac{4 \nu^{2}+1}{8 z} \sin \left(z-\frac{\pi}{4}\right)\right), \\
& \tilde{Y}_{\nu}(z) \approx \sqrt{\frac{2}{\pi z}}\left(\sin \left(z-\frac{\pi}{4}\right)-\frac{4 \nu^{2}+1}{8 z} \cos \left(z-\frac{\pi}{4}\right)\right) .
\end{aligned}
$$

Using $(\mathrm{A}-6)$ and $(\mathrm{A}-7)$ in $(14)$, we obtain the following expression

$$
\begin{aligned}
J_{i \nu}(z) & \approx \cosh \left(\frac{\pi}{2} \nu\right) \sqrt{\frac{2}{\pi z}}\left(e^{i\left(z-\frac{\pi}{4}\right)}\left[1-i \frac{4 \nu^{2}+1}{8 z}\right]\right) \\
& \approx \cosh \left(\frac{\pi}{2} \nu\right) \sqrt{\frac{2}{\pi z}}\left(e^{i\left(z-\frac{4 \nu^{2}+1}{8 z}-\frac{\pi}{4}\right)}\right),
\end{aligned}
$$

and the analytical solutions become Eqs. (19) and 20.

\section{Non-relativistic limit of the KG solutions in an expanding well}

In order to show that Eq. 20 reduces to the solution of the Schrödinger equation (21) in the non-relativistic limit, we need to examine the imaginary exponential of Eq. 20. What does the argument $z-\frac{4 \nu^{2}+1}{8 z}$ become in the non-relativistic limit? The terms coming from $z$ are equal to

$$
\frac{L_{0}}{\lambda_{C} \beta}+\frac{m c^{2} t}{\hbar}-\frac{m v x^{2}}{2 \hbar L}+\ldots
$$

from which we recover the term proportional to $x^{2}$ that we have in (21). The terms coming from the other part are

$$
-\left(\frac{n^{2} \pi^{2}}{2 \beta^{2}}+\left(\frac{1}{8}-3 n^{2} \pi^{2}\right)+\ldots\right) \frac{1}{z}
$$

where we have only approximated the first part, $\left(4 \nu^{2}+\right.$ $1) / 8 \approx \frac{n^{2} \pi^{2}}{2 \beta^{2}}+\frac{1}{8}-\frac{1}{3} n^{2} \pi^{2}$. When $\beta$ is small, the dominant term is therefore $-\frac{n^{2} \pi^{2}}{2 \beta^{2} z}$. If we do a Taylor expansion of $\frac{1}{z}$ in $\beta$, we find that $-\frac{1}{\beta^{2} z}=$

$$
\begin{aligned}
& -\frac{\lambda_{C}}{L_{0} \beta}+\frac{c \lambda_{C} t}{L_{0}^{2}}+\frac{\lambda_{C}}{2 L_{0}^{3}}\left(-2 c^{2} t^{2}-x^{2}\right) \beta \\
& +\frac{c \lambda_{C} t}{2 L_{0}^{4}}\left(2 c^{2} t^{2}+3 x^{2}\right) \beta^{2} \\
& +\frac{\lambda_{C}}{8 L_{0}^{5}}\left(-8 c^{4} t^{4}-24 c^{2} t^{2} x^{2}-3 x^{4}\right) \beta^{3}+(\ldots)
\end{aligned}
$$

The first term can be absorbed in the normalization factor, we factorize $\frac{c \lambda_{C} t}{L_{0}}$ in the resulting expression $\left.(A-11)+\frac{\lambda_{C}}{L_{0} \beta}\right)$ and we keep only the terms with leading powers of $c$. Doing that we get

$$
\frac{c \lambda_{C} t}{L_{0}}\left(\frac{1}{L_{0}}-\frac{c t}{L_{0}^{2}} \beta+\frac{c^{2} t^{2}}{L_{0}^{3}} \beta^{2}-\frac{c^{3} t^{3}}{L_{0}^{4}} \beta^{3}+(\ldots)\right),
$$

which is the Taylor expansion of $c \lambda_{C} t /\left(L_{0} L\right)=$ $\hbar t /\left(m L_{0} L\right)$ hence the term $e^{-i \frac{\hbar n^{2} \pi^{2} t}{2 m L_{0} L}}$ that we have in 21 .

\section{Validity of the approximations and numerical simulations}

If the parameter $\frac{\nu^{2}}{z}$ is very small, then the approximation $\mathrm{A}-8$ should be very good. In order to evaluate the accuracy of this approximation, we have plotted the real and imaginary parts of the quantity

$$
\frac{J_{i \nu}(z)}{\cosh \left(\frac{\pi \nu}{2}\right) \sqrt{\frac{2}{\pi z}}}-e^{i\left(z-\frac{4 \nu^{2}+1}{8 z}-\frac{\pi}{4}\right)},
$$

respectively denoted by $d_{1}$ and $d_{2}$, for a given $\nu(100 \pi)$ and for various $z$. Contrary to what we would expect intuitively, the approximation becomes worse for some critical value of $X=\log _{10}(z)$; at about $X=12.3$, there is a sudden increase in $d_{1}$ and $d_{2}$, from about $10^{-7}$ to $10^{-4}$. This is presumably due to round off errors affecting the validity of numerical routines. Therefore, if we plan on using the approximations, we must choose our parameters (mass, initial box length and so on) in such 
a way as to have the lowest absolute error for the Bessel functions (say something of the order of $10^{-7}$ ).

To avoid any problem of interpretation of the KleinGordon equation, we work in the non-relativistic regime characterized by

$$
\beta \ll 1 \quad \text { and } \quad z \approx \frac{L_{0}}{\lambda_{C} \beta} \gg 1 .
$$

Furthermore the approximation for the Bessel function $J_{-i k_{1}}(z)$ (used in $\Psi_{-, 1}(t, x)$ ) will be valid provided that

$$
\frac{k_{1}^{2}}{z} \approx \frac{\pi^{2}}{\beta^{2} z} \approx \frac{\pi^{2} \lambda_{C}}{\beta L_{0}} \ll 1 .
$$

Finally we don't want $n_{\max }$ (the maximum index for the reduced basis) to be too large, otherwise the numerical integration needed to obtain the $c_{n}$ (see Eq. (34) ) would not be feasible. To estimate $n_{\max }$, we need to estimate the number of oscillations in $\Psi_{-, 1}(t=0, x)$ between $x=$ 0 and $x=L_{0}$. From the expression $(20)$, we find that it is given by

$$
\frac{z(0,0)-z\left(0, L_{0}\right)}{2 \pi} \approx \frac{\beta L_{0}}{4 \pi \lambda_{C}} .
$$

We see that the last two conditions are antagonistic: we can't have a very good approximation of $\Psi_{-, 1}(t=0, x)$ by (20) unless $n_{\max }$ is very large. This explains why a numerical simulation using the approximations for the Bessel functions would be very precise only if $n_{\max }$ is very large. 